\title{
Highlights in Neutrino and Astroparticle Physics
}

\author{
G. Giacomelli ${ }^{\mathrm{a}}$ \\ ${ }^{a}$ Dipartimento di Fisica dell'Università di Bologna and INFN, Sezione di Bologna, \\ viale Berti Pichat 6/2, I-40127 Bologna, Italy \\ e-mail: giacomelli@bo.infn.it
}

Closing lecture of the 1999 S. Miniato Workshop, Neutrino and Astroparticle Physics, S.Miniato, Italy, 17-21 May 99.

The main highlights from the papers presented at this workshop are briefly reviewed and discussed in a general context.

\section{INTRODUCTION}

This was a timely workshop, because of the many new important experimental results on neutrino physics and astrophysics, and the renewed interest in astroparticle physics, both experimentally and theoretically.

The experimental evidences on neutrino oscillations are mounting, and many new experiments are being planned to definitely prove these indications and accurately measure the neutrino oscillation parameters.

The astrophysical $\gamma$-ray bursts seem to be a dominant phenomenon in our universe. New, larger and more sophisticated detectors are planned to study the higher energy $\gamma$-rays.

Neutrino astrophysics started in the 1960's with the first detection of electron neutrinos from the sun, and was in a sense reborn in 1987 with the detection of electron antineutrinos from $\mathrm{Su}$ pernova SN87A. Many detectors are now ready to study these two phenomena. High energy muon neutrino detectors of large volumes are entering the scene, possibly opening the field of high energy muon neutrino astronomy.

Many search experiments are trying to detect possible components of the Dark Matter (DM), or search for new particles predicted by the Standard Model (SM) of particle physics, and by theories which go beyond the SM.

The study of the highest energy cosmic rays is another field of interest, in particular for de- terminig the mechanisms responsible for their acceleration. Very large area detectors, above and below ground, are needed to study this field.

A Large effort is being made to develop instrumentation for the detection of gravitational waves, which should reveal some of the most violent phenomena occurring in the cosmos. The detectors include supercooled antennas at $0.1 \mathrm{~K}$ and very long interferometers (few $\mathrm{km}$ ).

In this summary of the workshop I shall recall many of the papers presented, with special emphasis on neutrino oscillations and on higher energy phenomena; I shall not be able to cover in detail all subjects, nor quote all results. I apologize for this impossibility and for possible omissions.

During the workshop we were informed of the sudden death of Bianca Monteleoni Conforto, a colleague and a collaborator. I dedicate these notes to her memory.

\section{NEUTRINO PHYSICS}

Most of the interest in this field concentrated on experimental results relevant to neutrino oscillations.

\subsection{Atmospheric neutrinos}

The interest in atmospheric neutrinos has grown in the last year, after the Neutrino '98 Conference in Takayama, Japan. New, higher statistics data have been presented by the Soudan 2 [1], MACRO [2], and SuperKamiokande (SK) [3] [if] 
collaborations. The measured flux of muons induced by atmospheric $\nu_{\mu}$ shows a reduction with respect to the expectation; the reduction depends on the neutrino energy and direction. For $\nu_{e}$ induced electrons there is no deviation from the prediction. The three experiments can explain the $\nu_{\mu}$ reduction in terms of $\nu_{\mu} \rightarrow \nu_{\tau}$ neutrino oscillations, with maximum mixing and $\Delta m^{2}$ values of few times $10^{-3} \mathrm{eV}^{2}$.

In the simplest scenario of two flavor oscillations, the survival probability of a pure $\nu_{\mu}$ beam is

$P\left(\nu_{\mu} \rightarrow \nu_{\mu}\right)=1-\sin ^{2} 2 \theta \sin ^{2}\left(\frac{1.27 \Delta m^{2} \cdot L}{E_{\nu}}\right)$

where $\Delta m^{2}=m_{2}^{2}-m_{1}^{2}$ is the mass difference of the two neutrino mass states, $\theta$ is the mixing angle, $E_{\nu}$ is the neutrino energy and $L$ is the path length from the $\nu_{\mu}$ production point to the detector. For atmospheric neutrinos $L$ can be estimated through the neutrino arrival direction $\Theta$. For upgoing neutrinos, as the zenith angle $\Theta$ changes, one has $L \sim 2 R_{\oplus} \cdot \cos \Theta\left(R_{\oplus}\right.$ is the Earth radius), while $L$ is only few tens of kilometers for vertical downgoing neutrinos.

Atmospheric neutrinos are detected in the $\mathrm{Su}-$ perKamiokande (SK) water Cerenkov detector via their interactions with $\mathrm{p}$ and ${ }^{16} \mathrm{O}$ nuclei in the $22500 \mathrm{~m}^{3}$ water fiducial volume $\left(50.000 \mathrm{~m}^{3}\right.$ total volume). Three different classes of events are defined (with increasing average energy of the parent neutrino): fully contained events (FC), partially contained events (PC) and upward-going muons. FC events are further subdivided into sub-GeV and multi-GeV. Electrons are identified in the FC sample. The zenith angle distribution for e-like sub-GeV and multi-GeV events are in reasonable agreement with the predictions assuming no-oscillations. Instead the $\mu$-like events deviate considerably from the prediction, see Fig. 1. The ratio of the measured numbers of muons to electrons normalized to the respective Monte Carlo predictions is affected by a smaller systematic error, and it enhances the anomaly [屯].

Assuming two flavor oscillations Fig.2 shows the $90 \%$ C.L. contours delimiting the accepted regions by Kamiokande and SK. The SK data favour $\nu_{\mu} \rightarrow \nu_{\tau}$ neutrino oscillations with $\Delta m^{2}=$
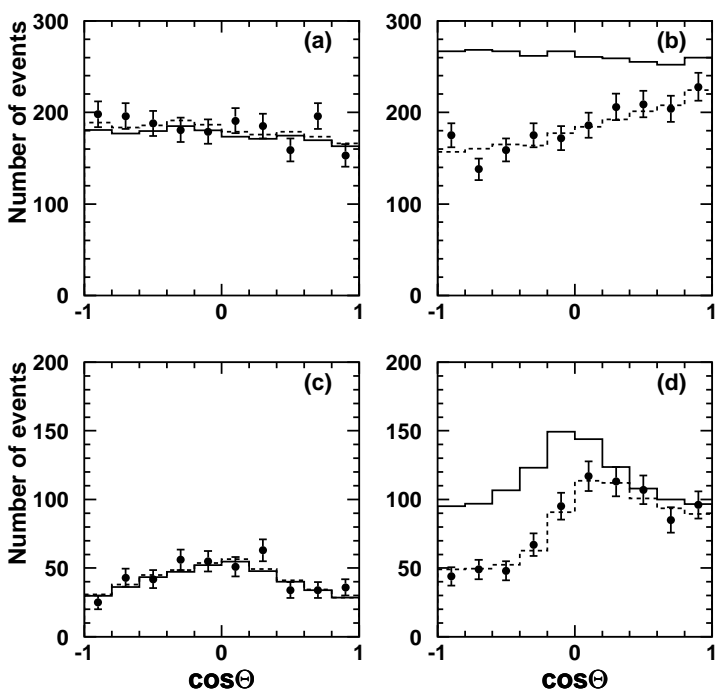

Figure 1. SK data. Zenith angle distributions of sub$\mathrm{GeV}$ (a) $e$-like events and (b) $\mu$-like, (c) multi-GeV $e$-like, (d) multi $\mathrm{GeV} \mu$-like data. Upward going particles have $\cos \theta \lesssim 0$. The black points are the data, the solid lines are the Monte Carlo expectations for nooscillations; the dashed lines are the MC predictions for $\nu_{\mu} \rightarrow \nu_{\tau}$ oscillations with $\Delta m^{2}=3.510^{-3} \mathrm{eV}^{2}$ and $\sin ^{2} 2 \theta=1$ [3][4].

$(1.5-6) 10^{-3} \mathrm{eV}^{2}$ and $\sin ^{2} 2 \theta>0.9$. For more details on further data on upthroughgoing muons and stopping muons, see the paper presented by Kajita at this workshop [4], and [3]. SK obtains also indications for an east-west asymmetry, which may be considered a confirmation of the flux calculations and of the experimental methods 顿.

The Soudan 2 results support the oscillation hypothesis by measuring atmospheric $\nu_{\mu}$ and $\nu_{e}$ interactions at low energies, below $1 \mathrm{GeV}$ [1]. A different detection technique (drift chamber calorimeter) is used in this case; the total mass of the detector is about $1 \mathrm{kt}$ and the total exposure is $4.6 \mathrm{kt} y$. Fig. 3 shows, for the high-resolution contained data, vs $\log \left(\mathrm{L} / \mathrm{E}_{\nu}\right)$ relative to the nooscillation expectations. Still with low statistical significance, the data agree with a reduction of $\nu_{\mu}$ events compared to expectations, while the $\nu_{e}$ events agree with expectations.

The MACRO detector is a box of $76.6 \mathrm{~m} \times 12 \mathrm{~m} \times 9.3 \mathrm{~m}$ located 


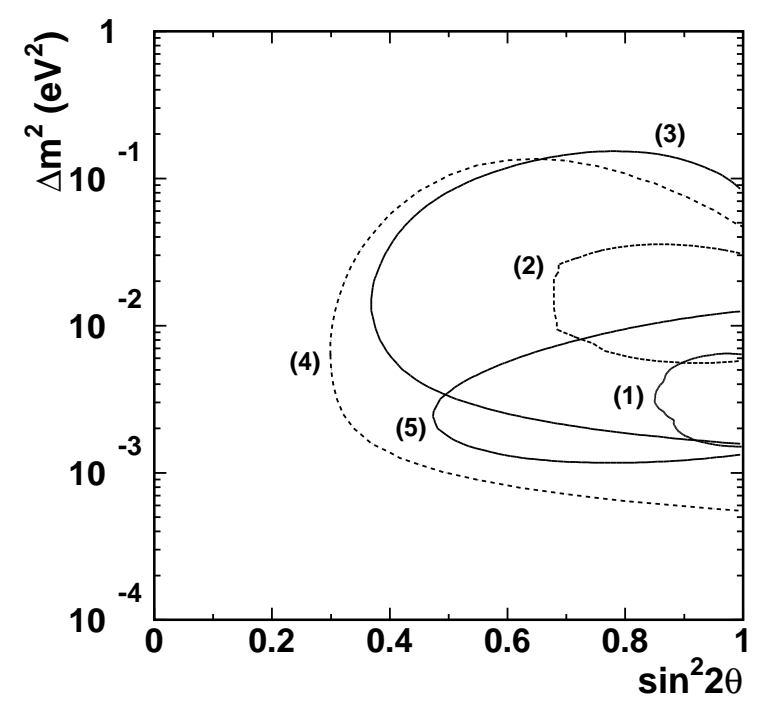

Figure 2. The $90 \%$ C.L. contours in the $\sin ^{2} 2 \theta$ and $\Delta m^{2}$ plane for $\nu_{\mu} \rightarrow \nu_{\tau}$ oscillations for the Kamiokande and SuperKamiokande data [4]. (1) and (2) refer to contained events from SK and Kamiokande, respectively. (3) and (4) refer to upward through-going muons from Super-K. and K. (5) shows the region obtained by the (stopping/throughgoing) ratio of upward going muons from SK.

at the Gran Sasso Lab.; the detection elements are planes of limited streamer tubes for tracking and liquid scintillation counters for timing. The lower half of the detector is filled with trays of absorbers alternating with streamer tube planes, while the upper part is open. The angular resolution for muons achieved by the streamer tubes is $<1^{\circ}$. The time resolution of the scintillators is $0.5 \mathrm{~ns}$. Fig. 1 shows the measured topologies.

The up throughgoing muons come from $\nu_{\mu}$ with $\bar{E}_{\nu_{\mu}} \simeq 80 \mathrm{GeV}$ interacting in the rock below the detector; their flight direction is determined by time-of-flight (t.o.f.). $\nu_{\mu}$ with $\bar{E}_{\nu_{\mu}} \simeq 4 \mathrm{GeV}$ interact inside the lower apparatus; yielding upgoing muons (IU). The partially contained downgoing events (ID) and upward going stopping muons (UGS) are identified via topological constraints.

Monte Carlo simulations play a crucial role in atmospheric neutrino studies. Macro used the Bartol neutrino flux 5 and the deep inelastic

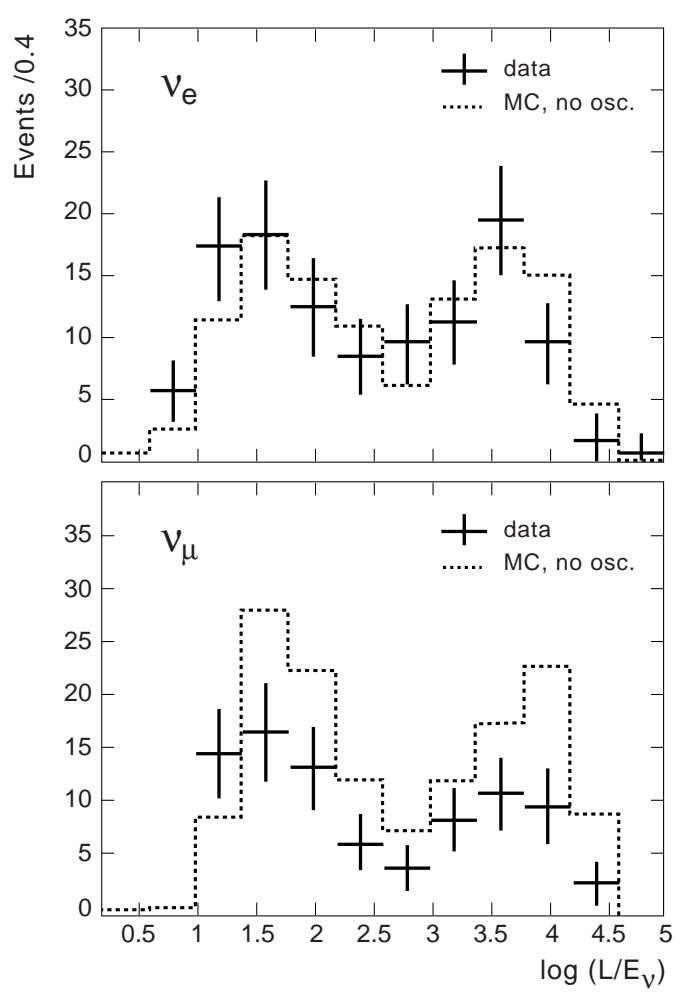

Figure 3. Soudan 2 results on the number of observed $\nu_{e}$ (top) and $\nu_{\mu}$ (bottom) events as function of $L / E_{\nu}$. Only statistical errors are shown; the dashed lines are the MC predictions assuming no-oscillations. Note the lack of $\nu_{\mu}$ events at $\log _{10}(\mathrm{~L} / \mathrm{E}) \simeq 1.8$ and 3.9.

scattering (DIS) parton distribution of ref. [6] for the neutrino cross-sections. The propagation of muons is done using the energy loss calculations of ref. [7] in standard rock. The systematic uncertainty on the expected flux of muons is $\pm 17 \%$; this is a scale factor, that changes little the shape of the angular distribution.

The ratio of the observed to expected number of upthroughgoing muons is $0.74 \pm 0.031_{\text {stat }} \pm$ $0.044_{\text {sys }} \pm 0.12_{\text {theo }}$. Fig. 5 shows their zenith angle distribution compared to Monte Carlo expectation without neutrino oscillations (solid line); the dashed line is the fit to the data assuming $\nu_{\mu} \rightarrow \nu_{\tau}$ oscillations. The reduction in the detected number of events and the deformation of the zenith angle distribution may be due to $\nu_{\mu}$ 


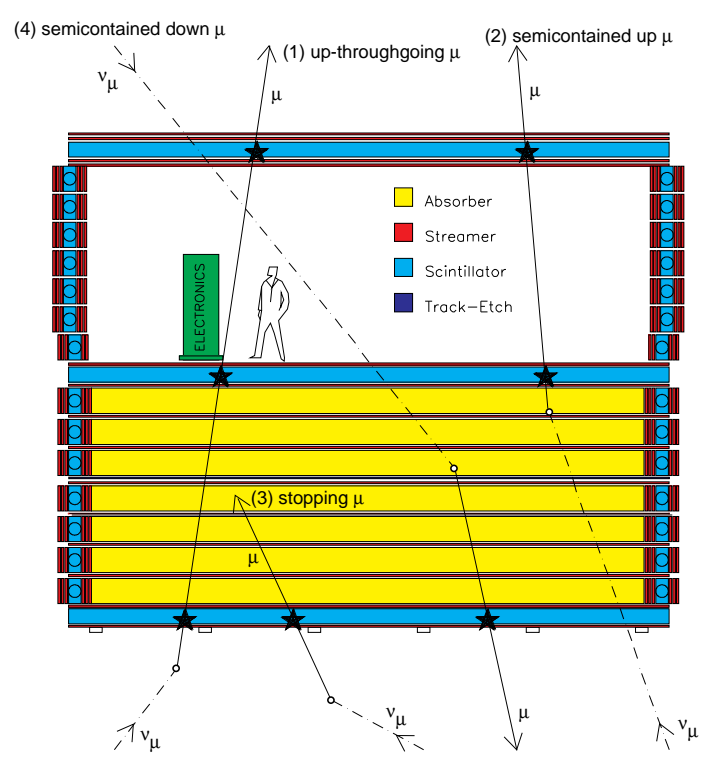

Figure 4. Sketch of different event topologies induced by muon neutrino interactions in or around MACRO. The stars represent the liquid scintillator hits.

disappearance: fewer events are expected near the vertical $(\cos \Theta=-1)$ than near the horizontal $(\cos \Theta=0)$, due to the longer path length of neutrinos from production to observation. The maximum of the $\chi^{2}$ probability corresponds to $\Delta m^{2}=$ $2.5 \times 10^{-3} \mathrm{eV}^{2}$ and maximum mixing. The confidence region at the $90 \%$ C.L. in $\left(\sin ^{2} 2 \theta, \Delta m^{2}\right)$ for $\nu_{\mu} \rightarrow \nu_{\tau}$ oscillations agrees and is somewhat larger than that of SK [2]. Notice the possible excess of events at $\cos \Theta \sim-0.65$ (also the upthroughgoing muons of SK have a similar hint); it is consistent with a statistical fluctuation, but it could be a hint for a more complex scenario.

Fig. 6 shows the zenith angle distribution of the semicontained (IU) and upstopping muons plus partially contained downgoing muons (UGS + ID). The data are within errors consistent with a constant deficit with respect to the MC expectations. The ratios of the number of observed to expected events are $R_{I D+U G S}=\left(\frac{\text { Data }}{M C}\right)_{I D+U G S} \simeq$ 0.71 and $R_{I U} \simeq 0.57$. The theoretical and systematic errors are largely reduced (from $25 \%$ to about $5 \%$ ) if the ratio of ratios is considered, $\mathcal{R}=R_{I U} / R_{I D+U G S}=0.80 \pm 0.09_{\text {stat }}$; for no oscillations one expects $R=1$. The reductions are consistent with $\nu_{\mu} \rightarrow \nu_{\tau}$ oscillations with maxi-

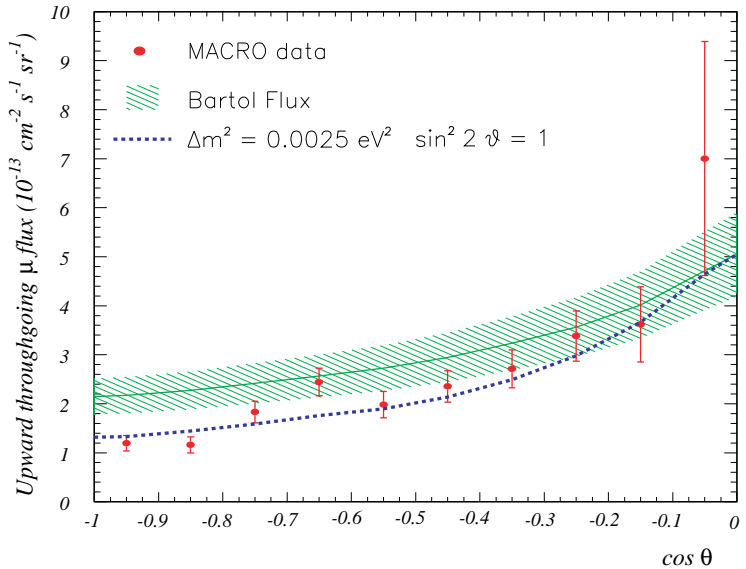

Figure 5. Measured flux (points) of upward throughgoing muon $v s$. the cosine of zenith angle $\Theta$. The solid line is the prediction with no oscillations; the $17 \%$ scale uncertainty is shown as the dashed region, the error on the shape is almost negligible. The dashed line shows the prediction assuming two-flavor neutrino oscillations.

mum mixing and $\Delta m^{2} \sim 10^{-3} \div 10^{-2} e V^{2}$.

Several theoretical papers tried to interpret the atmospheric neutrino data in terms of oscillations among three neutrino types; the differences with the simpler $\nu_{\mu}-\nu_{\tau}$ possibility are small [8]. Other authors considered $\nu_{\mu} \rightarrow \nu_{\text {sterile, }}$, which is slightly disfavoured by the data. Others include $\nu_{e}, \nu_{\mu}, \nu_{\tau}, \nu_{\text {sterile }}$.

G. Battistoni [9] discussed the effects of the approximations used in the Monte Carlo predictions. Present MCs use the collinear approach, which cannot be a good approximation at low energies. But 3-D effects are smeared out because of Fermi motion of the nucleons in nuclei. Uncertainties remain in the knowledge of primary cosmic ray spectra, secondary particle production and neutrino cross sections. Hopefully measurements of muons in the atmosphere 10] could improve the predictions, though the sub-GeV range remains problematic.

Improved atmospheric neutrino detectors are under discussion 11 .

\subsection{Solar neutrinos}

Solar $\nu_{e}$ come from a chain of nuclear reactions and decays in the centre of the sun. The three important components of the spectrum are: 

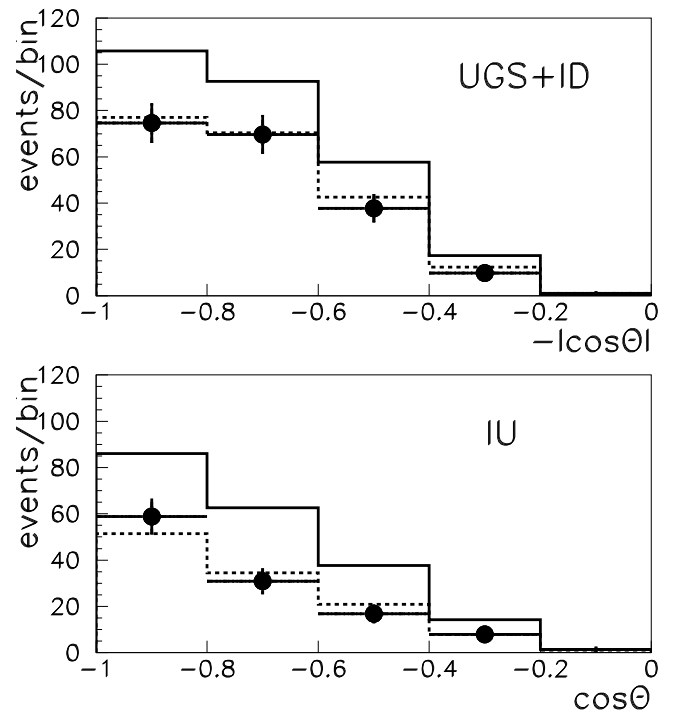

Figure 6. MACRO Zenith angle distributions for (ID+UGS) and IU events. The data (black points with error bars) are compared with the Monte Carlo expectations assuming no oscillations (full lines) and two-flavour oscillations (dashed lines) using maximum mixing and $\Delta m^{2}=2.5 \times 10^{-3} \mathrm{eV}^{2}$.

i) the energetic neutrinos from ${ }^{8} B$ decay; ii) the monoenergetic neutrinos from ${ }^{7} \mathrm{Be}+e^{-} \rightarrow{ }^{7} \mathrm{Li}+\nu_{e}$ $\left(\mathrm{E}_{\nu_{e}}=0.862 \mathrm{MeV}\right)$ and iii) the low-energy part, the $p p$ neutrinos $\left(\mathrm{E}_{\nu_{e}} \leq 0.41 \mathrm{MeV}\right)$ (most abundant).

Experimental measurements of solar neutrinos have been performed by five experiments using three different reactions 12 .

The first measurement of solar neutrinos used a radiochemical method via inverse $\beta$ decay, $\nu_{e}+{ }^{37} \mathrm{Cl} \rightarrow{ }^{37} \mathrm{Ar}+e^{-}$, which has a neutrino energy threshold $\mathrm{E}_{\nu t h}=814 \mathrm{keV}$. The experiment, sensitive to ${ }^{7} \mathrm{Be}$ and ${ }^{8} \mathrm{~B}$ neutrinos yields a flux smaller than that predicted by the Standard Solar Model (SSM): $\left(\phi_{7_{B e}}+\phi_{8_{B}}\right)(\mathrm{Cl})<$ $\left(\phi_{7_{B e}}+\phi_{8_{B}}\right)(\mathrm{SSM})$.

The second measurement was performed in the Kamiokande water Cerenkov detector using the reaction $\nu_{e}+e^{-} \rightarrow \nu_{e}+e^{-}$. They apply a cut at $\mathrm{E}_{\nu t h} \simeq 7 \mathrm{MeV}$ and are thus sensitive only to ${ }^{8} B$ neutrinos. The angular distribution is peaked in the direction of the sun and therefore confirms that the detected neutrinos come from the sun. They obtained a ratio expected $/$ measured $=0.417 \pm 0.069$. Combining this with the chlorine result, one has a discrepancy expressed as $\left(\phi_{7_{B e}}+\phi_{8_{B}}\right)(\mathrm{Cl})<\phi_{8_{B}}(\mathrm{Ka})$.

A third reaction is studied by radiochemical methods using ${ }^{71} \mathrm{Ga}$ in metallic (SAGE) and in a hydrochloric water solution (GALLEX, GNO): $\nu_{e}+{ }^{71} \mathrm{Ga} \rightarrow{ }^{71} \mathrm{Ge}+e^{-}$, which has a threshold at $\mathrm{E}_{\nu_{e}}=233 \mathrm{keV}$. Thus one may measure the neutrinos coming from the $p p$ reactions, proving that the sun is a $p p$ nuclear fusion plant. The experiments yield values smaller than the SSM prediction; these low values and the comparison with the preceeding measurements, lead to $\phi_{7_{B e}}($ meas $)<\phi_{7_{B e}}(\mathrm{SSM})$, which seems to be the main problem.

Superkamiokande presented at this workshop new results on solar neutrinos which further confirm the above statements. The data also confirm that the solar $\nu_{e}$ come from the sun [4], see Fig. [7. A day-night effect might have been observed by SK: this would be expected for the MSW effect. Also a seasonal variation due to the eccentricity of the earth orbit might have been observed.

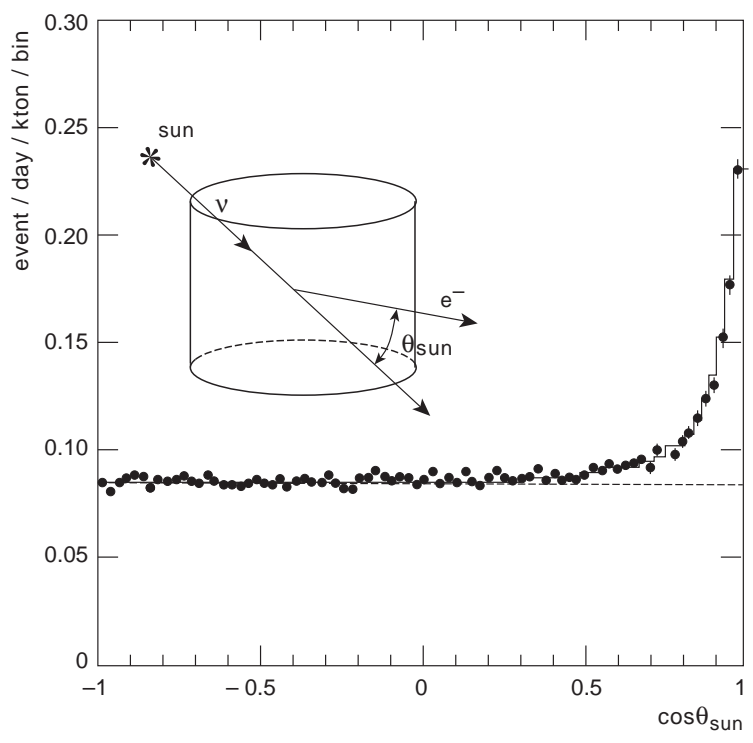

Figure 7. Data from SuperKamiokande on the arrival direction of solar neutrinos measured via the reaction $\nu_{e} e^{-} \rightarrow \nu_{e} e^{-}$. Notice the peak at $\cos \theta=1$, towards the sun. 
The lack of observed Be solar neutrinos seems at present to be the essence of the solar neutrino problem. This could be due to a faulty experiment. Assuming that the experiments are correct, physicists looked for an astrophysical solution and at neutrino oscillations. Improvements have been made in the knowledge of the sun interior [12] and it seems that one cannot explain the deficits. One is therefore left with the possibility of neutrino oscillations.

Possible solutions of the problem assuming neutrino oscillations in vacuum and possible solutions assuming neutrino oscillations in solar matter (the MSW effect) are indicated in the compilation of Fig. 8; more up to date compilations have been presented in [11]-[13]; the MSW low-mixing solution seems to be disfavoured.

Most solar neutrino experiments are relatively low-rate experiments. Superkamiokande and future experiments have higher rates and have more specific aims. The SNO detector in Sudbury, Canada is starting to take data with neutrino interactions in $D_{2} O$. At Gran Sasso the Borexino experiment plans to detect ${ }^{7} \mathrm{Be}$ neutrinos via the reaction $\nu_{e} e^{-} \rightarrow \nu_{e} e^{-}$in liquid scintillators 12]; ICARUS should detect $\nu_{e}$ interactions in an ${ }^{40} \mathrm{Ar}$ TPC [17]. There are discussions about the possible use of $\mathrm{Li}$ I $(\mathrm{Eu})$ scintillation counters.

\subsection{Accelerator and reactor experiments}

We have heard numerous reports about short baseline accelerator experiments: LSND, KARMEN, NOMAD, CHORUS and the future MINIBOONE. LSND gave a possible signal for neutrino oscillations for $\Delta m^{2} \sim 1 \mathrm{eV}^{2}$ and $10^{-3} \lesssim$ $\sin ^{2} 2 \theta<$ few $10^{-2}$, see Fig. 8 [13]. The other experiments gave limits, which are globally summarized in Fig. 8. Several technical improvements were made by these experiments; I shall only recall the revival of the emulsion technique for neutrino physics, specifically for $\nu_{\tau}$ detection, because of the exceptional space resolution $(\sim 1 \mu \mathrm{m})$ of the technique [14].

The results from the Chooz and Palo Verde reactor experiments exclude $\nu_{e} \rightarrow \nu_{\mu}$ oscillations for $\Delta m^{2}>10^{-3} \mathrm{eV}^{2}$ and $2 \times 10^{-2}<\sin ^{2} 2 \theta<1$, not shown properly in Fig. 8 .

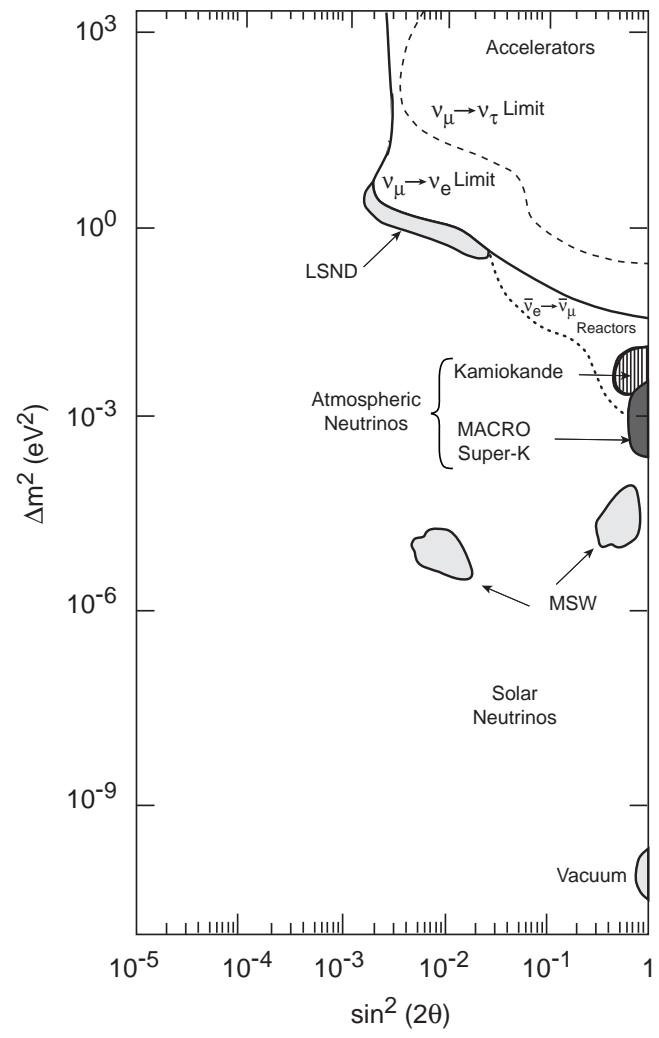

Figure 8. Compilation of excluded regions by short baseline accelerator and reactor experiments, and of allowed regions by the LSND accelerator experiment [13], by the atmospheric $\nu_{\mu}$ experiments and by the solar neutrino experiments, assuming MSW matter oscillations and vacuum oscillations. More updated compilations are given in [4], [12]-[18].

\subsection{Long baseline experiments}

Several long baseline experiments have been proposed; they will cover the region $\Delta m^{2}>$ $10^{-3} \mathrm{eV}^{2}$ and $\sin ^{2} 2 \theta>10^{-2}$ [15] 18].

- K2K: from KEK to SuperKamiokande $(230 \mathrm{~km})$ is starting to take data; they also have a near detector 陆.

- MINOS: from the Fermilab main injector to the Soudan mine $(730 \mathrm{~km})$ is under construction; it is a 6 kton calorimeter detector; they will also have a near detector 15.

- KAMLAND: $\bar{\nu}_{e}$ from nuclear reactors will be detected in the Kamiokande mine with a liquid scintillator detector 16 .

- ICARUS $\rightarrow$ now ICANOE: from CERN-SPS 
to Gran Sasso $(730 \mathrm{~km})$ [17]; ICARUS will be an "electronic" bubble chamber; NOE a tracking calorimeter detector.

- OPERA: from CERN to Gran Sasso 18]; it is basically a (large) emulsion detector.

\subsection{Direct measurement of the $\bar{\nu}_{\mathbf{e}}$ mass}

Tritium decay, $t \rightarrow^{3} \mathrm{He}+e^{-}+\bar{\nu}_{e}$ with $\mathrm{Q}=$ $18.6 \mathrm{keV}, t_{1 / 2}=12.3 \mathrm{y}$, has been used by many groups to obtain increasingly better limits on the $\bar{\nu}_{e}$ mass $\left(\simeq \bar{\nu}_{1}\right.$ mass if there is small mixing $)$. The techniques and the calculations have been constantly improved. The latest results with the improved Mainz set up give a 95\% C.L. upper limit $m_{\bar{\nu} e} \lesssim 2.8 \mathrm{eV}$ [19].

\subsection{Neutrinoless double beta decay}

For even-even nuclei the chain decays

$$
\begin{gathered}
(A, Z) \rightarrow(A, Z+1)+e^{-}+\bar{\nu}_{e}, \\
(A, Z+1) \rightarrow(A, Z+2)+e^{-}+\bar{\nu}_{e}
\end{gathered}
$$

are forbidden by energy conservation; the decay may be possible in a single step:

$$
\begin{gathered}
(A, Z) \rightarrow(A, Z+2)+2 e^{-}+2 \bar{\nu}_{e}, \\
(A, Z) \rightarrow(A, Z+2)+2 e^{-}+x, \\
(A, Z) \rightarrow(A, Z+2)+2 e^{-}
\end{gathered}
$$

The neutrinoless double beta decay, Eq. (2d), is forbidden by lepton number conservation; it would be allowed if $\nu_{e}$ and $\bar{\nu}_{e}$ were identical and if they had a non-zero mass. The energy spectrum for the sum of the energies of the two electrons, $E=E_{1}+E_{2}$, is different for each of the three cases: a line for (2d), a continuum peaked at low $E$ for (2b) and a continuum peaked at higher $E$ for $(2 \mathrm{c})$.

Most of the direct searches for neutrinoless double beta decays use materials which act both as source and detector, such as ${ }^{76} \mathrm{Ge} \rightarrow{ }^{76} \mathrm{Se}+2 e^{-2}$ 20. Germanium detectors ranging from 1 to $7 \mathrm{~kg}$ have been used. Normal germanium contains $15 \%{ }^{76} \mathrm{Ge}$. The Heidelberg-Moscow Collaboration uses several kilograms of enriched germanium containing $85 \%{ }^{76} \mathrm{Ge}$. From an exposure of $24 \mathrm{~kg} y$ they quote $t_{1 / 2}>6 \times 10^{25} y(90 \% C L)$, which in certain models corresponds to $m_{\nu_{e}}<$ $0.2 \mathrm{eV}$. Some groups use visual detectors, separating the spatial detection of the two electrons. The double beta decay ${ }^{136} \mathrm{Xe} \rightarrow{ }^{136} \mathrm{Ba}+2 e^{-}$has a favorable transition energy of $2479 \mathrm{keV}$.

Considerable work is going on in the development of cryogenic detectors for double beta decays and for dark matter searches [20]. At low temperature the heat capacity is very small, and a small energy deposition implies a relatively large increase in temperature. Four cryogenic crystals of $\mathrm{Te} \mathrm{O}_{2}$, each about $340 \mathrm{~g}$, were used by the Milano-Gran Sasso collaboration to study the double beta decay of ${ }^{130} \mathrm{Te}$. Four sapphire detectors, each of $262 \mathrm{~g}$, are used by the CRESST experiment in a search for dark matter WIMPs.

\section{NEUTRINO ASTROPHYSICS}

\subsection{Neutrino Astronomy}

One of the main interests in neutrino astronomy is connected with the great penetrating power of neutrinos, which allows us to look directly at their sources. The universe is filled with fossil low-energy neutrinos from the Big Bang. Low-energy neutrinos of $\sim 1 \mathrm{MeV}$ come continuously from the interior of stars like the sun; slightly-higher-energy neutrinos $(\sim 12$ $\mathrm{MeV}$ ) come in bursts from supernovae explosions. High-energy neutrinos $(>1 \mathrm{GeV})$, may come from non-thermal point sources. Neutrinos of $>1 \mathrm{GeV}$ may also come from the sun and the earth, where annihilations of WIMPs could take place.

\subsubsection{Neutrinos from stellar gravitational collapses}

Massive stars, $m>6 m_{\odot}$, evolve as increasingly heavier nuclei are produced and then burnt at their centres in a chain of thermonuclear processes, ultimately leading to the formation of a core composed of iron and nickel. When the core mass exceeds the Chandrasekar limit, the core implodes in a time slightly longer than the freefall time and leads to the formation of a neutron star. The energy released during a stellar collapse is at least the gravitational binding energy of the residual neutron star, $E \simeq 3 \times 10^{53}\left(\mathrm{~m} / \mathrm{m}_{\odot}^{2}\right)$ $(10 \mathrm{~km} / \mathrm{R}) \mathrm{ergs}, \simeq 10^{53} \mathrm{ergs} \simeq 0.1 \mathrm{~m}_{\odot}$, mostly in the form of neutrinos with $\left\langle E_{\nu}\right\rangle \simeq 12 \mathrm{MeV}$. About $4 \times 10^{57}$ neutrinos of each species are emitted. Three stages of neutrino emission may be 
identified.

All types of neutrinos may be detected via neutral current interactions with electrons, $\nu_{e} e^{-} \rightarrow$ $\nu_{e} e^{-}, \bar{\nu}_{e} e^{-} \rightarrow \bar{\nu}_{e} e^{-}$, etc, with a cross section $\sigma=1.7 \times 10^{-44} \mathrm{E}_{\nu}\left(M e V c m^{2}\right)$. The dominant reaction, $\bar{\nu}_{e} p \rightarrow n e^{+}$, with $\sigma=7.5 \times 10^{-44} E_{\nu}^{2}$ $\left(\mathrm{MeV}^{2} \mathrm{~cm}^{2}\right)$, is energetically possible only on free protons, as in $\mathrm{H}_{2} \mathrm{O}$ and in $\mathrm{C}_{n} \mathrm{H}_{2 n+2}$ detectors. The positron produced annihilates immediately, $e^{+} e^{-} \rightarrow 2 \gamma$, whilst the neutron is moderated and captured after a mean time of about $180 \mu \mathrm{s}$ $\left(n p \rightarrow d \gamma\right.$, with $\left.E_{\gamma} \sim 2.2 \mathrm{MeV}\right)$. The SNO detector with $D_{2} O$ will also detect $\nu_{e} n \rightarrow p e^{-}$. Because of the dependence of the cross section on neutrino energy, the average $e^{+}$energy is about $2 \mathrm{MeV}$ larger than the average $\bar{\nu}_{e}$ energy.

Only Supernova SN1987A in the Large Magellanic Cloud was observed with neutrinos. No other burst of supernova neutrinos has been detected. Present and future neutrino detectors, will only be able to observe galactic supernovae. An optimistic estimate of the rate of type-II Supernovae in our galaxy is one every $10-20$ years. Several detectors are kept alive all the time and a worldwide supernova watch is in operation.

\subsubsection{High-energy neutrino astronomy}

High-energy muon neutrinos can be detected via their charged-current interactions inside a detector or in the rock surrounding the detector leading to upgoing muons. Upward-going muons can be seen directly in Cerenkov detectors and can be separated by time-of-flight from downward-going muons in scintillators. At very high energies the $\nu_{\mu}-\mu$ angle is small and the effective target may be large. In order to observe celestial "point" sources of high-energy neutrinos one should plot for each muon its declination versus right ascension. A celestial source would reveal itself as an excess of events (in a certain direction) above the atmospheric neutrino background. Now, people are also looking at time coincidences with $\gamma$-ray bursts.

Several underground experiments performed searches for astrophysical sources of $\nu_{\mu}$, with negative results. In order to establish a flux limit for a specific source one may consider an error circle corresponding to the resolution of the detector in that direction ( $\simeq 3^{\circ}$ for tracking detectors, considerably more for $\mathrm{H}_{2} \mathrm{O}$ Cerenkov detectors), determine the number of events in that circle, and subtract the corresponding number of events expected from atmospheric neutrinos. MACRO, with about 1000 muon events, quotes limits at the $10^{-14} \mathrm{~cm}^{-2} \mathrm{~s}^{-1}$ level [21].

Neutrino telescopes. Much larger detectors, the so called Neutrino Telescopes, will be required to really attack the field of $\nu_{\mu}$ astronomy. Prototypes of neutrino telescopes may be considered the Cerenkov detectors NESTOR, ANTARES and NEMO under deep sea water, Baikal under lake water and AMANDA under ice at the South Pole 222. The final detector will be around $1 \mathrm{~km}^{3}$ of water or ice.

\subsection{Searches for WIMPs}

Weakly Interactive Massive Particles (WIMPs) could be part of the galactic dark matter. WIMPs should be neutral particles which may form a dissipationless gas trapped in the gravitational field of our Galaxy. Suitable WIMP candidates should have lifetimes comparable to the age of the Universe. In SUSY models, like the MSSM and SUGRA, they may be identified with the lightest neutralino; it is ok if $\mathrm{R}$ parity is violated provided it leads to a long lifetime neutralino. WIMPs have been searched for by direct and indirect methods.

- Direct searches. WIMPs may be searched for via their interactions in refined low energy detectors of $10-100 \mathrm{~kg}$ mass. The WIMPs scatter elastically with the nuclei of the detector, with cross sections of the order of the weak ones or smaller. A scattering leads to a recoil of few $\mathrm{keV}$ energy. The detectors must have low radioactivity, be well shielded and use electronics which reduces unwanted noise signals. The DAMA collaboration presented results obtained with a $100 \mathrm{~kg}$ $\mathrm{NaI}(\mathrm{Tl})$ detector, looking for a signal modulated over a one year period. They find a probable signal which could correspond to a neutralino mass of $50-60 \mathrm{GeV}$ [23].

- Indirect methods. WIMPs could be intercepted by celestial bodies, slowed down and trapped in their centres. WIMPs and anti-WIMPs could annihilate and yield neutrinos of $\mathrm{GeV}$ or $\mathrm{TeV}$ en- 
ergies. The neutrinos would travel and interact below the detector yielding high energy muons which can be detected. The search should be performed in small angular windows around the directions of the celestial bodies. The 90\% C.L. MACRO limit for the flux from the Earth centre is $\sim 10^{-14} \mathrm{~cm}^{-2} \mathrm{~s}^{-1}$ for a $10^{\circ}$ cone around the vertical, see Fig. 9 [21]. For the same cone searched for around the Sun direction, the limit stands at $\sim 1.4 \times 10^{-14} \mathrm{~cm}^{-2} \mathrm{~s}^{-1}$.

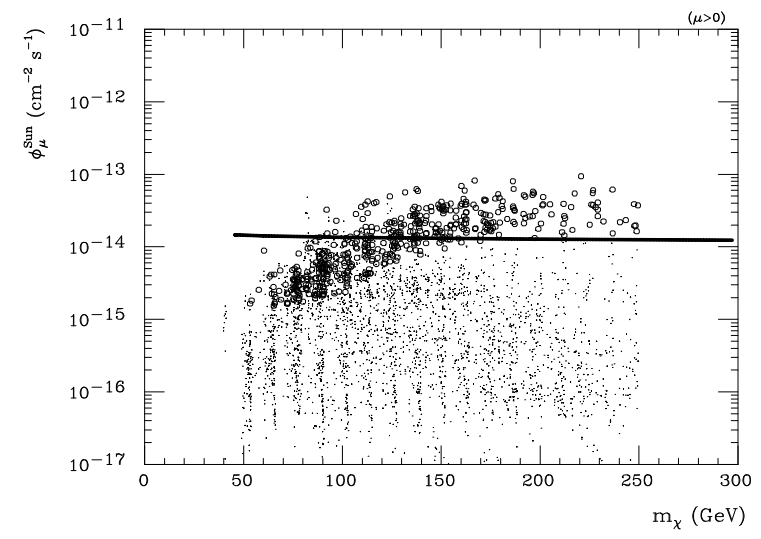

Figure 9. Upward-going muon flux vs neutralino mass $m_{\chi}$ from the Earth. Each dot is obtained varying model parameters, leaving $\mu>0$. Solid line: MACRO flux limit (90\% C.L.); the limit for the nooscillation hypothesis is indistinguishable in the log scale from the one for the $\nu_{\mu} \rightarrow \nu_{\tau}$ oscillations hypothesis. The open circles indicate models excluded by direct measurements and assume a local dark matter density of $0.3 \mathrm{GeV} \mathrm{cm}{ }^{-3}$. The DAMA indication is at $m_{x} \simeq 50-60 \mathrm{GeV}$.

\section{HIGH ENERGY COSMIC RAYS}

The all-particle spectrum of cosmic rays is shown in Fig. 10 24.

\subsection{Underground muons}

Underground experiments detect a sizeable downward flux of high-energy muons, single and multiple, coming from high-energy cosmic rays. Muons reaching the Gran Sasso detectors traverse a minimum path length of 3100 m.w.e. and an average one of 3700 m.w.e.. A muon must therefore have an energy larger than $1.3 \mathrm{TeV}$ to reach the

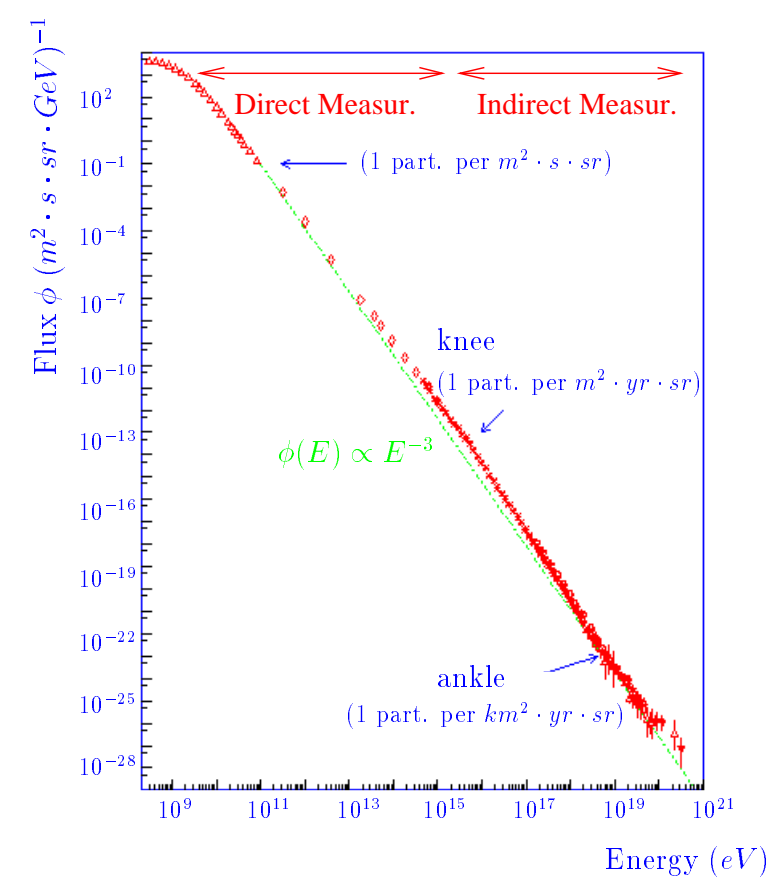

Figure 10. All particle energy spectrum of primary Cosmic Rays.

detectors. The muon distribution in local coordinates (azimuth $\varphi$ and zenith $\theta$ ) reflects the shape of the mountain: it may be considered an $\mathrm{x}$-ray photograph of the mountain. Experiments proved that the arrival time distribution of underground muons is random.

The vertical muon flux $I(h)$, where $h$ is the slant depth, was measured with increasing accuracy by several experiments. The flux may be represented by

$$
I(h)=B\left(h_{1} / h\right)^{2} e^{-h / h_{1}}
$$

with $B=(1.81 \pm 0.06) \times 10^{-6} \mathrm{~cm}^{-2} \mathrm{~s}^{-1} \mathrm{sr}^{-1}, \quad h_{1}=$ $(1231 \pm 1) \mathrm{hg} \mathrm{cm}^{-2}$. The muon surface flux, obtained from the measured underground muon flux, is $d N_{\mu} / d E d \Omega=A E^{-\gamma}$ with $\gamma \simeq 2.78$.

Seasonal variations. Selected muon data from several experiments were used to search for seasonal variations. The muon rate shows clear variations of about $\pm 1.4 \%$ amplitude which repeats over the years. The muons come from pion 
and kaon decays in the upper atmosphere (at depths of less than 200 mbar); their intensity becomes greater when the atmosphere is warmer. A new measurement performed by AMANDA at the South pole exhibits a much larger effect $(\sim \pm 15 \%)$ reflecting the 6 month darkness and 6 month light [22]. From underground muons it is possible to measure the effective temperature of the higher atmosphere to about $1{ }^{\circ} \mathrm{C}$ !

Muon astronomy. In "muon astronomy" one assumes that high-energy muons remember the arrival direction of the parent high-energy particle with the hope that the parent particle has not deviated. Thus a search may be made for celestial point sources, d.c., periodic or episodic. The interest in muon astronomy started in 1985 with reports of an excess of underground muons from the direction of Cyg. X-3 and with the Cyg. $\mathrm{X}-3$ periodicity. Some reports of muon excesses could be connected with intense radio flares. In order to exclude with certainty a variation of the muon flux from the direction of Cyg. $\mathrm{X}-3$ one has to analize data over a long period of time. Upper limits for a d.c. signal were established for specific sources, Cyg. X-3, Her X1, 1E2259+59, and the Crab. The d.c. limits range from 3 to $6 \times 10^{-13} \mathrm{~cm}^{-2} \mathrm{~s}^{-1}$ 21]. For Cyg. X-3, MACRO searched for a muon signal modulated by the 4.8 $\mathrm{h} \mathrm{X}$-ray period. The phase diagram does not exhibit any excess above background in any phase bin. The upper limit on a modulated sygnal is $F_{\text {mod }} \leq 3 \times 10^{-13} \mathrm{~cm}^{-2} \mathrm{~s}^{-1}$.

Multiple muons. Multiple muons carry infomation about the energy spectrum and the chemical composition of primary cosmic rays with energies $\geq 50 \mathrm{TeV}$. The sensitivity to composition arises from the fact that heavy nuclei are more effective than protons in producing multiple muons. The measurable distributions are: i) The decoherence function (the distribution of the distance between two muons) 25. ii) The decorrelation function (the double-differential distribution of two muon relative angles). iii) The multiplicity distribution. iv) The muon group sub-structure.

The analyses require a model of the hadronic interactions at high energy (nucleon-nucleus and nucleus-nucleus), trial models of the energy variation of the composition of cosmic rays, simulation

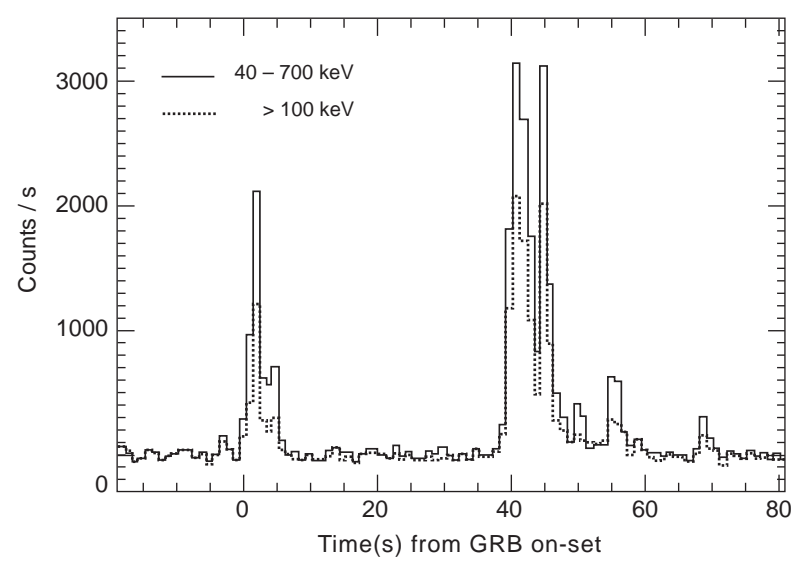

Figure 11. Examples of $\gamma$-ray bursts at x-ray energies (BeppoSAX).

of the cascade in air and in the rock, and a simulation of the detector. In practice one uses iteration procedures with continuous improvements in models and simulations, and eventually a multiparameter fit of all avaible data.

A slow increase of the average primary mass is observed when going from $10^{3}$ to $10^{4} \mathrm{TeV}$, i.e. when crossing the "knee" of the cosmic ray allparticle flux [25] [26].

It has to be noted that the muons in the same bundle arrive at the same time to within few $n s$.

\subsection{Cosmic rays of highest energies}

The origin of high-energy cosmic rays is essentially unknown, and it is difficult to devise acceleration mechanisms for the highest energy cosmic rays. Recently magnetic monopoles of relatively low mass accelerated by the galactic magnetic field to high energies and high velocites have been proposed as possible sources of the highest energy cosmic rays. It should be remembered that cosmic ray nuclei with energies $>4 \times 10^{19} \mathrm{eV}$ cannot come from distances $>50 \mathrm{Mpc}$ because of the Greisen cut-off caused by the interaction of protons with the $2.7 \mathrm{~K}$ photons of the cosmic backround radiation (at these energies the c.m. $p \gamma$ energy is above pion threshold, the cross section becomes large, and cosmic rays are soon degraded in energy).

It is clear that more data are needed and that this requires large Extensive Air Shower Arrays 
(EAS). We have heard reports from KASKADE and EASTOP 26. The largest new project is AUGER, with a first array in South America; a similar array will also be built in North America. Each Auger array will cover $\sim 5000 \mathrm{~km}^{2}$, with different types of detectors (hybrid air shower detectors): sampling water tanks and improved fly's Eyes detectors which detect the nitrogen luminescence in the atmosphere, thus measuring the shower profile 27.

Among the different types of proposed detectors for large arrays we have heard Sorel's presentation about the possibility of using standard solar pannels connected in series/parallel to detect the Cerenkov light 24.

\section{SCIENTIFIC EXPLORATION OF SPACE}

At this meeting we had a number of reports on physics and astrophysics research performed with balloons, satellites and the space station. There is an increasing effort in this field.

Recently balloon experiments have been performed to measure cosmic ray muons in the atmosphere [10]: these measurements are relevant for a more precise determination of the atmospheric neutrino flux.

The BeppoSAX satellite measured x-ray bursts, identifying $14 \mathrm{x}$-ray sources as the counterparts of $\gamma$-ray bursts (see Section 6) [28].

The AMS (Alpha Magnetic Spectrometer) experiment on the space station should make a thorough search for antimatter, measure the cosmic ray composition, and perform other searches. A test flight was successful and it has already provided important information on the flux of $p, d,{ }^{3} \mathrm{He},{ }^{4} \mathrm{He}$, and limits on antimatter 29.

A variety of experiments are becoming realities. For instance PAMELA is measuring $\bar{p}, e^{+}$of $100-200 \mathrm{GeV}$ and is making a search for $\overline{\mathrm{He}}$ nuclei 30. AGILE should be operative in 2002 [30]; it has several $\gamma$ ray detectors optimized to cover different $\gamma$ ray energies above $30 \mathrm{MeV}$, preliminary results have been obtained by NINA, etc. [30].

\section{6. $\gamma$-RAY BURSTS}

Since few years, the observation of $\gamma$-ray bursts (GRBs) poses one of the main misteries of astrophysics. The $\gamma$-ray observatory, on board the Compton satellite, observed every day, a new $\gamma$ ray burst of $\mathrm{MeV}$ energy. The burst durations are from $30 \mathrm{~ms}$ to 1000s (but this depends on the sensitivity and time resolution of the experiment: one may only see the tip of an iceberg). The bursts come from all directions of space; in almost all cases they represent a single episode. The rise time of the bursts is very fast, and this suggests that they could be connected with neutron stars. Measurements from the BeppoSAX satellite observe the GRBs at x-ray energies, Fig. 11. determining more accurately the position of the source; they see a tail in intensity (afterglow). When seeing a burst, BeppoSAX alerts the astronomical community; it was thus possible to observe the optical counterparts of x-ray emitters; the optical signal lasts a few days 28]; at least one appeared to be at the border of a far away galaxy. It should be stressed that, even if it is seen in $\mathrm{x}$ ray and in the visible, most of the emitted energy of GRBs is in $\gamma$ rays, at $\mathrm{MeV}$ energies.

It would be interesting to observe the GRBs at higher energies (multi $\mathrm{GeV}$ ) [31. It would even be more interesting to observe them with neutrinos; trials are being made, but probably one needs larger neutrino telescopes, with lower energy thresholds.

\section{RARE PARTICLES AND PROTON DECAY SEARCHES}

\subsection{Magnetic Monopoles}

Grand Unified Theories (GUTs) of electroweak and strong interactions predict the existence of magnetic monopoles (MMs) with large mass, larger than $10^{16} \mathrm{GeV}$, and magnetic charges $g=$ $n g_{\text {Dirac }}=n c / 2 e=n 68 e$, with $n=1,2, \ldots$ - These theories leave open the question of monopole abundance. MMs were probably produced in the early universe, at the end of the GUT era as point defects; others may have been produced in ultra-high energy collisions. Standard cosmology predicts too many monopoles, 
whereas models with inflation at the GUT phase transition predict very few. Several superstring models predict the existence of multiply-charged MMs $(n=3)$. In some models, the primordial monopoles appeared when the temperature of the universe reached relatively low values. These monopoles were probably not diluted by inflation in the early universe. The existence of large-scale magnetic fields, on the galactic scale, leads to an astrophysical constraint, the so-called Parker bound, with an upper limit on the monopole flux at the level of $10^{-15} \mathrm{~cm}^{-2} \mathrm{~s}^{-1} \mathrm{sr}^{-1}$; an extended Parker bound leads to a flux limit almost an order of magnitude smaller.

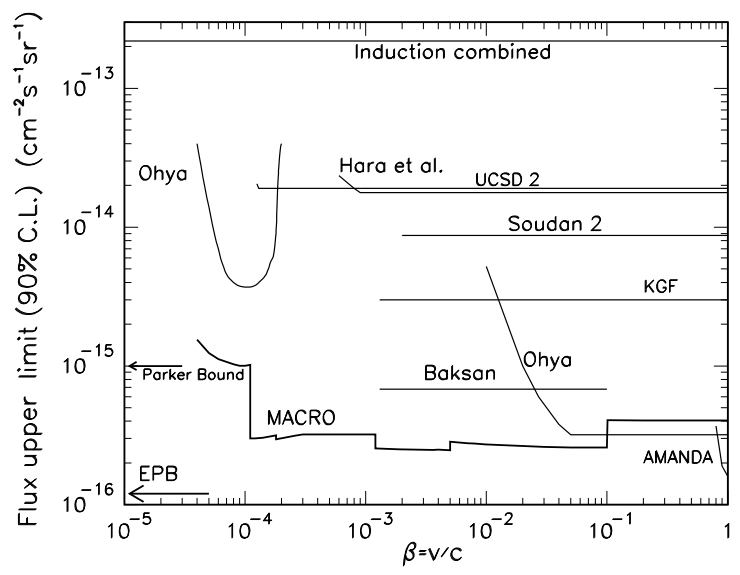

Figure 12. Compilation of $90 \%$ C.L. upper limits for a flux of heavy magnetic monopoles [32].

Underground experiments have searched for MMs in the penetrating cosmic radiation using scintillators, gas tubes, and nuclear track detectors via $d E / d x$, time of flight and pulse shape analyses. At present there are only a few large experiments. They tested the sensitivity of their detectors to low velocity MMs.

New limits have been presented by MACRO [32] and by AMANDA [22]. The present limits on massive cosmic MMs are summarized in Fig. 12 for $g=g_{D}$ bare poles with mass $>10^{16} \mathrm{GeV}$ and for catalysis cross sections smaller than few $\mathrm{mb}$.

\subsection{Dark Matter}

Analyses of the rotation curves of stars in galaxies, and of galaxies in clusters of galaxies prove (assuming the validity of Newton's law) that most of the matter is unseen: $\Omega_{v i s} \simeq$ $0.01, \Omega_{\text {halo }} \simeq 0.1, \Omega_{T H} \simeq 1$, where $\Omega=\rho / \rho_{c}$.

The unseen DM could be: a) baryonic, in the form of gas, planets like jupiter, brown dwarfs, nuclearites; b) non baryonic, i.e. a gas of particles. In the latter case there could be: i) hot DM, i. e. particles which were relativistic when in the early universe they decoupled from the rest of matter and radiation (an example could be neutrinos with a mass of a few eV); ii) cold DM, i.e. particles which were non-relativistic at decoupling (for example the WIMPs, see Section 3.2). These particles are probably located in the galactic halos; their abundance in the vicinity of the solar system could typically be $\sim 0.3 \mathrm{GeV} / \mathrm{cm}^{3}$, and their velocity $\sim 300 \mathrm{~km} / \mathrm{s}$.

Nuclearites. The hypothesized stable phase of quark matter, called strange quark matter or nuclearites, formed by quarks $u, d$ and $s$, may be the true ground state of QCD. Nuclearites could have masses ranging from a few $\mathrm{GeV}$ to the mass of a neutron star. Because of this wide range, searches were performed using a variety of experimental techniques. At this meeting new results have been presented, using techniques developed for MM searches. Limits for nuclearites with masses larger than $0.1 \mathrm{~g}$ (which can penetrate the earth) are at the level of the limits of MMs [32]; the limits are twice as large for nuclearites with $\mathrm{m}<0.1 \mathrm{~g}$, which cannot traverse the earth.

Other "exotic" objects, like the Q-balls (aggregates of squarks, sleptons and Higgs fields) have also been discussed at this meeting [32].

\subsection{Proton decay}

GUTs place quarks and leptons in the same multiplets. Quark $\longleftrightarrow$ lepton transitions are thus possible and should be mediated by supermassive vector bosons $X, Y$ with $m \sim 10^{14} \mathrm{GeV}$. A free proton may decay as $N \rightarrow \ell^{+}+\operatorname{meson}(\mathrm{s})$ or $N \rightarrow \bar{\nu}+\operatorname{meson}(\mathrm{s})$. Proton decay, with a predicted lifetime of the order of $10^{31} \mathrm{y}$, motivated the construction of the first underground 
detectors with masses of the order of $1000 t$. Present detectors are either water Ĉerenkov detectors (SK) or tracking calorimeters (Soudan 2). Water detectors have larger masses and more free protons and may detect the sense of the track direction. Tracking calorimeters have a higher spatial resolution and a better $\pi / \mu$ separation at energies of about $200 \mathrm{MeV}$. Technical developments are being made towards a TPC type liquid chamber (ICARUS). A $3 t$ prototype works well and a $600 t$ module is under construction. Superkamiokande presented the following limits

$$
\left\{\begin{array}{lll}
\tau B R\left(p \rightarrow e^{+} \pi^{\circ}\right) & >1.6 \times 10^{33} y \\
\tau B R\left(p \rightarrow \bar{\nu} K^{+}\right) & >6.7 \times 10^{32} y
\end{array}\right.
$$

at $90 \%$ C.L. [33]; they rule out the simplest SU(5) GUT models.

\section{SELECTED RESULTS FROM AC- CELERATOR EXPERIMENTS}

LEP. The four experiments at the LEP positron-electron collider provided new improved precision values of the $Z^{\circ}$ lines shape. The $Z^{\circ}$ mass is now known with a precision of two parts in $10^{5}$, and has acquired the status of one of the three basic inputs of the Standard Model (SM) of particle physics. An important quantity derived from the line shape parameters is the number of light neutrino species which is now

$N_{\nu}=\left(\frac{\Gamma_{i n v}}{\Gamma_{l}}\right) /\left(\frac{\Gamma_{\nu}}{\Gamma_{l}}\right)_{S M}=2.9835 \pm 0.0083$, see Fig. 13; a direct method (the neutrino counting method) confirms this result [34].

From this determination one may deduce the amount of helium expected in primordial nucleosynthesis: one expects $24 \%$, in fair agreement with astrophysical data. The charged lepton universality is now established at the $0.1 \%$ level; the muon and the tau lepton appear more and more to be replicas of the electron. The increased energy of LEP (LEP2) allowed to study the reaction $e^{+} e^{-} \rightarrow Z^{\circ} \rightarrow W^{+} W^{-}$, proving the existence of the triple boson vertex, $Z^{\circ} W^{+} W^{-}$, and a precise measurement of the $W^{ \pm}$mass, which very likely will become one of the three inputs of the SM. LEP1 allowed a detailed study of QCD properties, in particular a precise determination of the strong coupling constant and of its variation with energy (LEP2 is showing that the variation con-

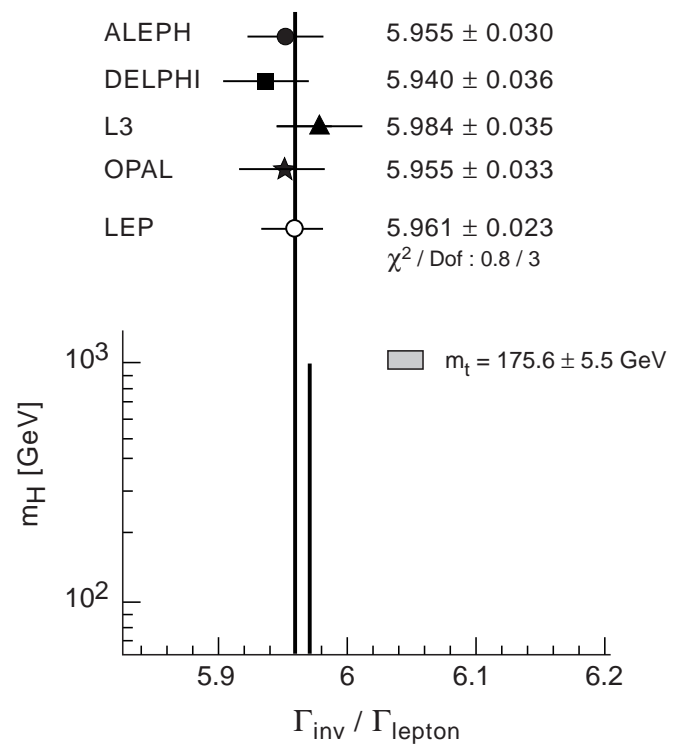

Figure 13. Ratio of the invisible width relative to the leptonic width at LEP. From this type of data, using updates, one obtains now that the number of neutrino families is $2.9835 \pm 0.0083$.

tinues to higher energies). It may be worth remembering that precision measurements lead to the first determination, below threshold, of the mass of the quark top and now gives a hint of the mass of the Higgs boson. LEP2 with data up to $\sqrt{s}=202 \mathrm{GeV}$ yields direct limits on the S.M. Higgs boson, $m_{H^{\circ}}>103 \mathrm{GeV}$, and on a variety of particles prediced by models beyond the SM.

HERA. At the asymmetric $e^{+} p$ collider $\left(E_{e}=\right.$ $26.7 \mathrm{GeV}, E p=820 \mathrm{GeV}$ ) at Hamburg, two experiments are providing a wealth of information on $\mathrm{CC}$ deep inelastic scattering, in particular at very small values of $x$ and large values of $Q^{2}$. They also measure the neutral current (NC) cross section, see Fig. 14 35]; notice that it is related to parton densities and takes into account effects of $x F_{3}$. A considerable part of the HERA program concerns the searches for particles predicted by models beyond the SM [35]. In particular new more stringent limits on leptoquarks have been presented. 


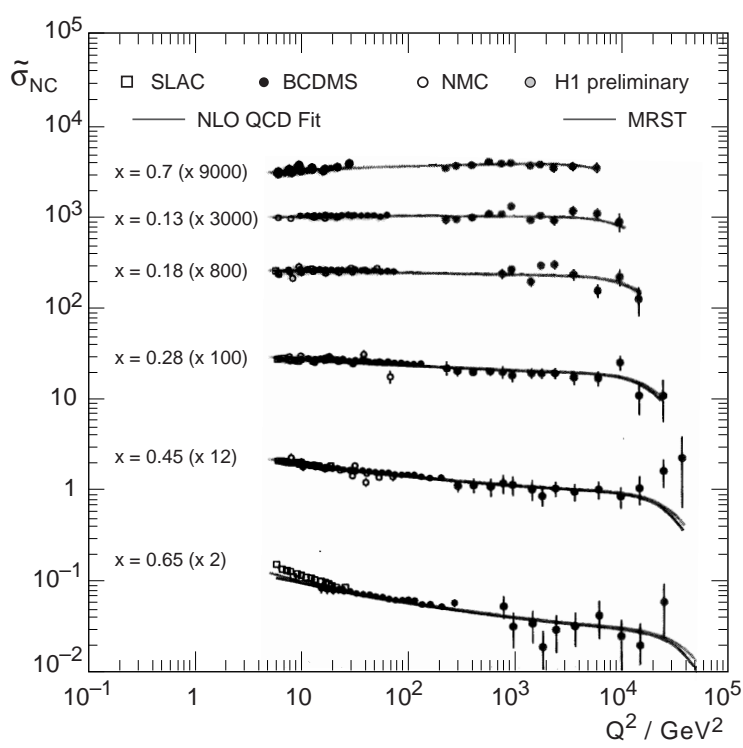

Figure 14. Neutral current reduced cross sections vs $Q^{2}$ measured in $e^{+} p$ collisions at HERA.

\section{GRAVITATIONAL WAVES}

The earth should be continously bombarded by gravitational waves produced by distant celestial bodies subject to "strong" gravitational effects. The amplitude of the gravitational wave emitted by a celestial body is proportional to its mass, to its acceleration, and to the inhomogeneity in its mass distribution. Gravitational waves are emitted when the quadrupole moment of an object of large mass is subject to large and fast variations. Only large celestial bodies subject to unusual accelerations should produce sizeable gravitational radiation measurable on earth. These bodies may be binary systems of close-by stars (in particular when a neutron star is about to fall on the other); they yield a periodic emission of gravitational waves, with frequencies from few hundred $\mathrm{Hz}$ to $1 \mathrm{MHz}$. Asymmetric supernovae explosions may give bursts of gravitational waves, with frequencies of the order of $1 \mathrm{kHz}$ over few ms. Also vibrating black holes, star accretions, galaxy formation, and the Big Bang may produce or have produced gravitational waves.

A gravitational wave is a transverse wave which travels at the speed of light. A gravitational wave should modify the distances between objects in the plane perpendicular to the direction of propagation of the wave. These deformations are expected to be extremely small. It has been estimated that a star collapse at the centre of our galaxy may produce a variation of the order of $h \sim 10^{-18}$ metre per metre of separation of two objects on earth. The Supernova 1987A in the large Magellanic Cloud could probably have produced a distortion 10 times smaller. A collapse in the Virgo cluster (at MPc), should yield relative variations of $10^{-21}$.

Very sensitive instruments are needed to observe gravitational waves. The two main lines developed until now are resonating bars at low temperatures and long laser interferometric systems. A major program is underway for both types of detectors, hoping to be able to detect gravitational star collapses up to the Virgo cluster, corresponding to $h \sim 10^{-21}$. The supercooled $(0.1$ K) bars NAUTILUS and AURIGA are operating in Frascati and Legnaro (Padova), respectively. A pair of long interefometers (LIGO) are under construction in the US, while one long interferometer is under construction at Pisa (VIRGO) [36]. Several detectors, in coincidence, are needed to ensure that the observed signal is not spurious.

The detection of gravitational waves would have far reaching consequences. It would prove the validity of the general theory of relativity; in astrophysics it would open up a new observational window related to violent phenomena in the universe.

\section{CONCLUSIONS}

We had an interesting and lively workshop on topical subjects. Many new interesting results were presented, as well as many new proposals: the field of astroparticle physics in general and of neutrinos in particular is very alive [37] and we look forward to many new exciting results in the future.

I would like to thank Ms. Luisa De Angelis for typing the manuscript. I acknowledge the cooperation of all the colleagues at the workshop and the help of the colleagues in Bologna, in particular of Dr. M. Giorgini. 


\section{REFERENCES}

1. Soudan 2 Collaboration, Proc. of NEUTRINO 98, Takayama, Japan; W.W.M. Allison et al., Phys. Lett. B449 (1999)137.

2. MACRO Collaboration, Proc. of NEUTRINO 98, hep-ex/9810008; M. Ambrosio et al. Phys. Lett. B434 (1998)451; hep-ex9904; INFN/AE-99/09, INFN/AE-99/10; M. Spurio, this workshop.

3. SuperKamiokande Collaboration, Proc. of NEUTRINO 98, hep-ex/9810001; Y. Fukuda et al., Phys. Rev. Lett. 81 (1998) 1562.

4. T Kajita, this workshop.

5. V. Agrawal et al., Phys. Rev. D53 (1996) 1314.

6. M. Glück et al., Z. Phys. C67 (1994) 433.

7. W. Lohmann et al., CERN-EP/85-03 (1985).

8. G. Fogli, S.F. King, D.P. Roy, W.G. Scott, this workshop.

9. G. Battistoni, this workshop.

10. M. Circella, this workshop.

11. F. Terranova, this workshop.

12. G. Bellini, A. Grandpierre, this workshop.

13. I. Stancu, this workshop.

14. T. Kawamura, this workshop.

15. P. Harris, this workshop.

16. G. Gratta (KAMLAND), this workshop.

17. C. Vignoli, this workshop.

18. A. Cocco, this workshop.

19. C. Weinheimer, this workshop.

20. E. Fiorini, Proc. of the Neutrino Telescope Workshop, Venezia (1999) page 35.

21. M. Ambrosio, et al., INFN/AE-99/11; INFN/AE-99/12, INFN/AE-99/13.

22. Neutrino telescopes: S. Bottai, C. Carlogam, P. Niessen, this workshop.

23. DAMA Collaboration, R. Barnabei et al., Phys. Lett. B450 (1999) 488.

24. M. Sorel, this workshop.

25. M. Sioli, E. Scapparone, this workshop.

26. K. Bernlöhr, A. Chiavassa, this workshop.

27. D. Zavrtanik, this workshop.

28. F. Frontera, A. Iyudin, this workshop.

29. A. Alpat, this workshop.

30. A. Morselli, R. Sparvoli, P. Spillantini, this workshop.

31. M. Iacovacci, this workshop.
32. L. Patrizii, M. Giorgini, M. Ouchrif, R. Wigmans, this workshop.

33. SuperKamiokande Collab., M. Shiozawa et al., Phys. Rev. Lett. 81 (1998) 3319; Y. Hayats et al., ICRR-453-99-11 (1999).

34. The LEP Collaborations, CERN-EP/99-15; M. Chemarin, this workshop.

35. M. Corradi, C. Beier, this workshop.

36. VIRGO, R. Passaquieti, this workshop.

37. Proceedings of the $5^{\text {th }}$ School on Non Accelerator Particle Astrophysics, Trieste University Press (1999). 